\title{
Identification of Genetic Variants Associated With Myocardial Infarction in Saudi Arabia
}

\author{
Kamal W. AI-Ghalayini, ${ }^{1}$ Mohammed A. Salama, ${ }^{2}$ Hadia Bassam Al Mahdi, ${ }^{2}$ Sameer Al-Harthi, ${ }^{3}$ \\ Wesam A. Alhejily, ${ }^{1}$ Mirvat A. Alasnag, ${ }^{7}$ Noura O. Tasbhji, ${ }^{2}$ Diana A.H. Al-Quwaie, ${ }^{4}$ Panos Deloukas, ${ }^{8}$ \\ Sherif Edris $2,5,6$ \\ ${ }^{1}$ Department of Internal Medicine, Faculty of Medicine, King Abdulaziz University, Jeddah, Saudi Arabia; ${ }^{2}$ Princess Al Jawhara \\ Albrahim Centre of Excellence in Research of Hereditary Disorders (PACER-HD), King Abdulaziz University, Jeddah, Saudi Arabia; \\ ${ }^{3}$ Department of Pharmacology, Faculty of Medicine, King Abdulaziz University, Jeddah, Saudi Arabia; ${ }^{4}$ Department of Biological \\ Sciences, Rabigh College of Science and Arts, King Abdulaziz University (KAU), Jeddah, Saudi Arabia; ${ }^{5}$ Department of Biological \\ Sciences, Faculty of Science, King Abdulaziz University, Jeddah, Saudi Arabia; ${ }^{6}$ Department of Biological Sciences, Science and Arts \\ College, Rabigh Campus, King Abdulaziz University (KAU), Jeddah, Saudi Arabia; ${ }^{7}$ Department of Cardiology, King Fahd Armed \\ Forces Hospital, Jeddah, Saudi Arabia; ${ }^{8}$ William Harvey Research Institute, Barts and The London School of Medicine and Dentistry, \\ Queen Mary University of London, London, UK
}

\section{ABSTRACT}

The genetic variants associated with various genetic disorders have not been identified decisively in Saudi Arabia. Among these variants, six known for their association with coronary artery disease or myocardial infarction (MI) were studied on Saudi patients. Reference single nucleotide polymorphisms (SNPs) of these variants are rs5174, rs11591147, rs2259816, rs111245230, rs3782886 and rs2259820, referring to genes LRP8, PCSK9, HNF1A, SVEP1, BRAP and HNF1A, respectively. The analysis employed polymerase chain reaction panel coupled with mini-sequencing (SNapShot multiplex system) in order to identify these variants. A total of $100 \mathrm{MI}$ patients and 103 healthy control individuals participated in this study. The six variants (SNPs) were evaluated for the risk of developing MI in the Saudi patients. Analysis of allele frequencies indicated that A allele of rs11591147 variant can be a protective allele, thus, is associated with the decreased risk of $\mathrm{MI}$ in Saudi individuals. Rare allele of rs111245230 variant (e.g., C allele) was extremely reduced, while rare allele of rs3782886 variant (e.g., $G$ allele) does not exist in the ethnic signature of the Saudi population. This study elucidates the possible prediction of risk factors associated with severe diseases in Saudi population utilizing SNapShot multiplex system.

\section{INTRODUCTION}

The prevalence of coronary artery disease (CAD) is increasingly observed worldwide. The World Health

Received March 8, 2019; accepted May 6, 2020.

Correspondence: SherifEdris, MD, Princess Al Fawhara Albrabim Centre of Excellence in Research of Hereditary Disorders (PACER-HD), King Abdulaziz University, Jeddab, Saudi Arabia; +966-593-66-23-84 (e-mail: sedris@aucegypt.edu).
Organization (WHO) anticipated that more than seven million people die from CAD each year in China [Wang 2014]. Myocardial infarction (MI) is the situation of the irreversible necrosis of the heart muscle that results from prolonged ischemia. Therefore, MI is considered the most crucial clinical manifestation of CAD [Matsuoka 2015]. The inheritance of assorted genetic variants functioning in association with environmental factors for promoting the disease status characterized myocardial infarction as a complex disease [Ferreira 2018]. More than $90 \%$ of the risk of an acute MI, in a casecontrolled study, is associated with the nine easily measured risk factors [Ma 2018]. The Chinese population was majorly affected by these risk factors, making a population of $89.9 \%$ at considerable risk.

The identification of several genetic loci has led to the progression of genome-wide association with CAD as well as MI risk [Isordia-Salas 2018]. Thereby, MI is a life-threatening disease as its pathogenesis is multifactorial and complex [Li 2019]. The development of MI has been demonstrated by the association between genetic factors, multiple genes, and environmental factors. Indeed, the inheritance of CAD indicates that $40-50 \%$ of the North Indian population are at risk of MI [Kashyap 2018].

The majority of the studied disease-related loci in European populations was replicated in populations of East Asian ancestry, however, this is not the case in Arab countries. Nikpay et al conducted a 1,000 genomes-based metanalysis by including a small number of South Asian (13\%) and East Asian (7\%) subjects [Nikpay 2015]. However, the study did not report on ethnic-specific effects. The direct effects of $\mathrm{CAD} / \mathrm{MI}$ risk alleles recognized in European populations on CAD risk in Asians generally were compatible, despite smaller data sets. On the other hand, there was a similar effect of size of CAD/MI risk alleles among the East Asian and European populations. In South Asia, the size effect apparently was weakened because of the interaction with unknown genetic or environmental factors along with the causal variants that may be inadequately tagged by markers present on available 

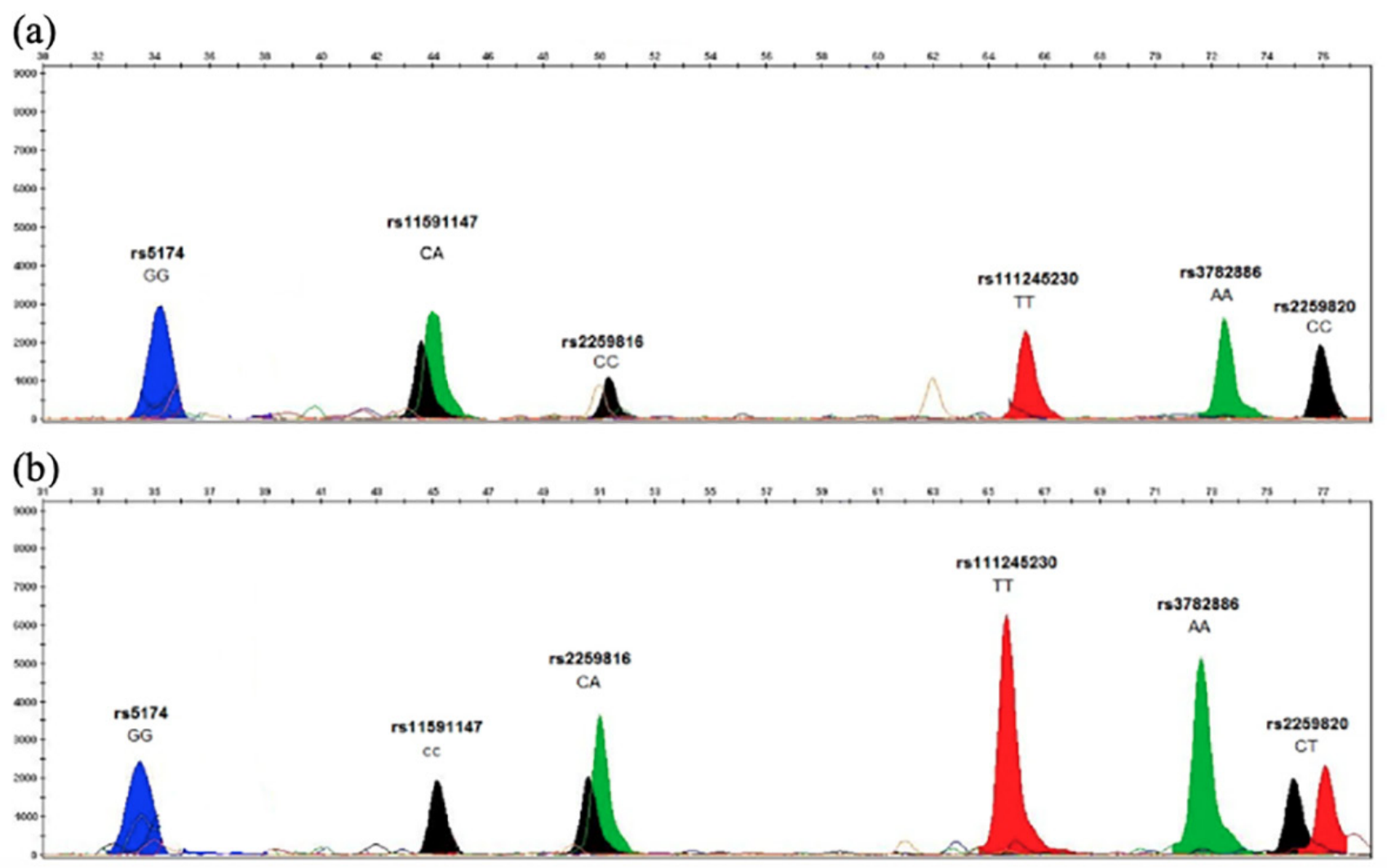

[FIGURE LEGEND MISSING]

genotyping arrays. This likely is to result in blunted genetic effects. Until recently, genetic variants associated with various disease-related genes have not been identified decisively in Saudi Arabia. Among them are the variants related to CAD or MI [Ferreira 2019; Kashyap 2018; Li 2019; Ma 2018]. Therefore, this study attempts to examine the possible association of genetic markers for susceptibility to MI among patients at King Abdulaziz University Hospital (KAUH) in Jeddah, Saudi Arabia. In addition, possible association of the ethnic background of Saudi individuals and any of these genetic markers also was investigated. The study includes screening the variants rs5174, rs11591147, s2259816, rs111245230, rs3782886 and rs2259820, referring to LRP8, PCSK9, HNF1A, SVEP1, BRAP and HNF1A genes, respectively.

\section{MATERIALS AND METHODS}

Blood collection: Peripheral blood samples were collected from $100 \mathrm{MI}$ patients and 103 healthy controls in $2 \mathrm{ml}$ EDTA-containing tubes, using a sterile syringe. Characteristics of subjects involved in the study in terms of numbers, gender, and smoking habits are shown in Table S1. The collected samples were transported in an ice container to the laboratory for further DNA isolation and stored at $-20^{\circ} \mathrm{C}$. Inclusion criteria involved (i) diagnosis of MI cases based on a history of MI, typical electrocardiographic change, left ventricular angiography, and coronary angiography; (ii) similar numbers of male to female, smokers to nonsmoker subjects; and (iii) subjects varied in age between 40 to 60 years old.

Genomic DNA isolation and quantification: The QIAamp DNA mini kit was used to extract DNA from the leukocytes of blood samples, while nanodrop-2000 spectrophotometer (Thermo Scientific, USA) was used to determine the isolated genomic DNA concentration.

SNaPshot Multiplex System for SNP genotyping: The DNA sequences encompassing six SNPs were selected from the genes LRP8, PCSK9, HNF1A (two SNPs), SVEP1, and BRAP. The ensemble (http://www.ensembl.org/index) and dbSNP (http://www.ncbi.nlm.nih.gov/ projects/SNP/) databases were used to select candidate polymorphic sites. Genes and reference SNP numbers along with nucleotide sequences of primers flanking the polymorphic sites and amplicon sizes used during PCR are described in Table 1.

Single base extension (SBE) primers subsequently were constructed to have lengths of 30 to 78 nucleotides by adding poly $\mathrm{dC}$ tails with varying lengths to secure the occurrence of six nucleotide differences in the SBE primer lengths, while avoiding regions of which other SNPs already had been described. SBE primer sequences and sizes along with the target variants of the consequent downstream SNPs are 
Table 1. PCR primers for the amplification of DNA fragments flanking six SNPs of five genes associated with myocardial infarction (MI)

\begin{tabular}{lccc}
\hline Gene Name & Variant & Forward $\left(5^{\prime} \rightarrow 3^{\prime}\right)$ & Reverse $\left(5^{\prime} \rightarrow 3^{\prime}\right)$ \\
\hline LRP8 & rs5174 & TGATCCAGAACAAACCCTAC & GGGTAGTCCATCATCTTCAA \\
PCSK9 & $r s 11591147$ & ACGGCCTCTAGGTCTCCT & CAGCAAGTCCCCAAAGAG \\
HNF1A & $r s 2259816$ & TATGGCCCTAAGAGTTTCAG & ATAGAGGTTGGCTGTCAATG \\
SVEP1 & $r s 111245230$ & AGCAGTAGGCAAGTCACATT & GACTCCTCACCCTCCTTATC \\
BRAP & $r s 3782886$ & GAGAATGAATAAAGCCATGC & GTCCATCACTTCCAGAAGAA \\
HNF1A & $r s 2259820$ & CATTGACAGCCAACCTCTAT & CCCACTAGCCTAGACAAAGA
\end{tabular}

Table 2. SBE primers used for the detection of six SNPs associated with myocardial infarction (MI)

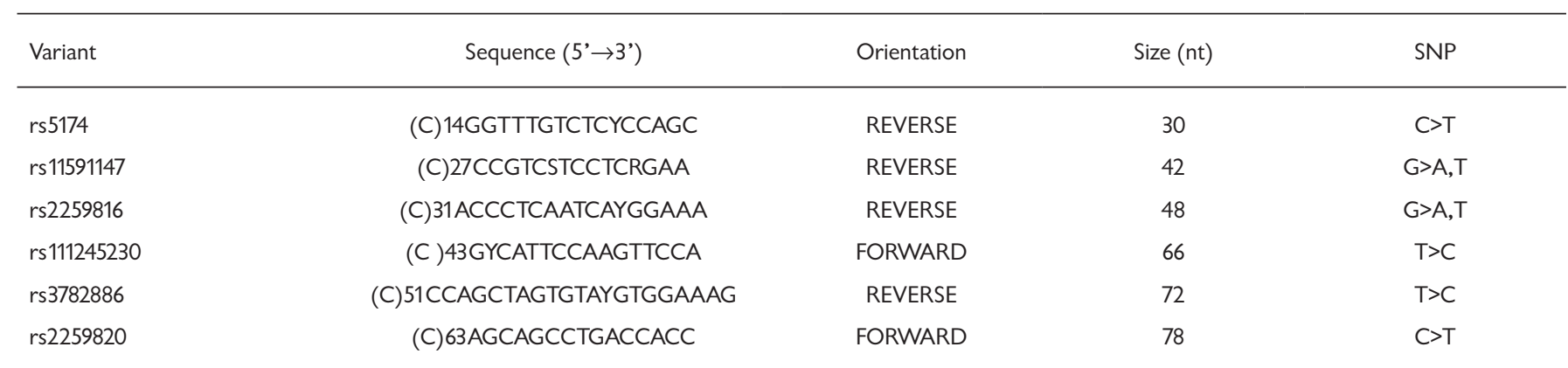

shown in Table 2.

Agarose gel electrophoresis: Gel electrophoresis was performed for the recovered PCR products using 3\% agarose gel with SYBR-safe DNA gel stain added to the gel to analyze the size and quality of the PCR products. Moreover, the gel was placed into UV Trans-illuminator to visualize the successive products.

SNaPshot reaction preparation: The SNaPshot reaction was performed by adding the six different single base extension (SBE) primers to be hybridized with the different corresponding PCR products and extended at the 3' ends by only one fluorescent labeled ddNTP, each with a different color. This reaction is called single base extension that is used in order to detect SNP alleles of the different six genes for participating individuals. SNaPshot analysis was conducted using the ABI Prism SNaPshot Multiplex kit (Applied Biosystems, Life Technologies, CA). An amount of 1 pmol of SBE primers $(1.5 \mu \mathrm{l})$ was added to an amount of $3.5 \mu \mathrm{l}$ of purified multiplexed PCR products $(30 \mathrm{ng})$ and $5 \mu \mathrm{l}$ of the ABI Prism SNaPshot ${ }^{\mathrm{TM}}$ Multiplex kit (Applied Biosystems, Life Technologies, CA) to reach a final reaction volume of $10 \mu l$. The SNaPshot reaction was placed on a Veriti ${ }^{\mathrm{TM}} 96$ well thermal cycler (Applied Biosystems, Life Technologies, CA) with the following conditions: 25 cycles of $96^{\circ} \mathrm{C}$ for $10 \mathrm{sec}, 48^{\circ} \mathrm{C}$ for $10 \mathrm{sec}$, and $60^{\circ} \mathrm{C}$ for $45 \mathrm{sec}$. Toward the end of the $\mathrm{SNaPshot}$ reaction samples were treated with 1 unit of shrimp alkaline phosphatase (GE Healthcare, Little Chalfont, UK) at $37^{\circ} \mathrm{C}$ for $60 \mathrm{~min}$ followed by $75^{\circ} \mathrm{C}$ for 15 min to inactivate the enzyme.

$\mathrm{SNaPshot}$ analysis: The purified $\mathrm{SNaPshot}$ reaction was, then, denatured at $95^{\circ} \mathrm{C}$ for $5 \mathrm{~min}$ in a Veriti ${ }^{\mathrm{TM}} 96$ well thermal cycler (Applied Biosystems Inc., Life Technologies, CA) after mixing with $1 \mu \mathrm{l}$ of, $9.5 \mu \mathrm{l}$ of Hi-Di formamide and $0.5 \mu \mathrm{l}$ of GeneScan ${ }^{\mathrm{TM}} \mathrm{LIZ120}{ }^{\mathrm{TM}}$ internal size standard (Applied Biosystems Inc., Life Technologies, CA). Then, fragments were analyzed in a 3500 Genetic Analyzer (Applied Biosystems Inc., Life Technologies, CA) using a POP-7 $7 \mathrm{TM}$ Polymer (Applied Biosystems Inc., Life Technologies, CA). Conditions during fragment analysis were as follows: injection $(15 \mathrm{sec})$, pre-run $(3 \mathrm{~min})$, run time $(15 \mathrm{~min})$, and data delay $(4 \mathrm{~min})$. The resulted patterns were analyzed using GeneMapper Software v4.1.

SNaPshot validation: DNA samples of 20 patients and 20 control individuals were genotyped for the six SNPs by Sanger sequencing. The results of direct sequencing should match those of SNaPshot analyses in order to confirm 100\% compatibility of the results.

Statistical analysis: The sequence variants were identified using the Bioedit software 6 editor version. The allele and genotype frequencies among the samples were estimated from the results of SNaPShot Assay. Hardy-Weinberg Equilibrium (HWE) was used to detect allelic frequencies and $\chi^{2}$ was used to detect significance of data among genotypic frequencies. A compact tool package for analysis and conversion of genotype data and SNP Tools for Microsoft Excel was used.

\section{RESULTS}

The aim of the study was to detect genetic markers related to MI among Saudi patients and the possible association of any of these markers with the ethnic signature 
Table 3. Genotypes and the numbers of the MI patients along with the healthy controls and the frequencies of the rare alleles of variants used in the study

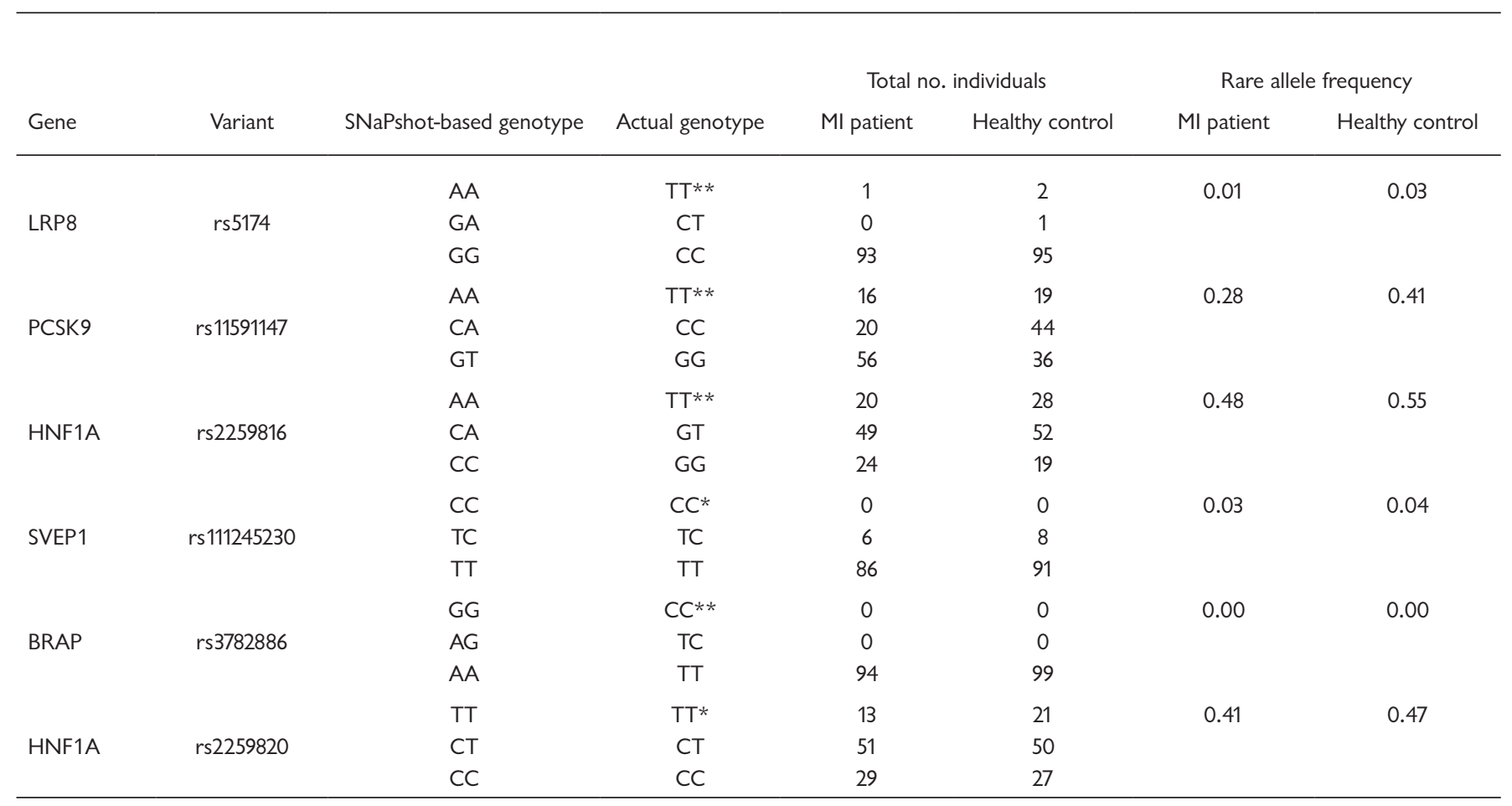

*Rare allele, **Complementary rare allele

Table 4. Results of chi square (X2) and odd ratio (OR) within the $95 \%$ confidence interval (Cl) of six selected variants

\begin{tabular}{|c|c|c|c|c|c|}
\hline rs5174 & 1.96 & 0.17 & 21.96 & 1.272 & .53 \\
\hline rs2259816 & 1.77 & 0.77 & 4.06 & 1.818 & .403 \\
\hline rs 111245230 & 0.95 & 0.06 & 15.35 & $N / A^{*}$ & $\mathrm{~N} / \mathrm{A}$ \\
\hline rs2259820 & 1.74 & 0.73 & 4.13 & 1.834 & .40 \\
\hline
\end{tabular}

*As calculation of one column is zero, $\chi^{2}$ cannot be computed

$\dagger$ As calculations of two columns are zero, $\chi^{2}$ cannot be computed

of Saudi participants. These markers included the variants rs5174, rs11591147, s2259816, rs111245230, rs3782886 and rs2259820, referring to LRP8, PCSK9, HNF1A, SVEP1, BRAP and HNF1A genes, respectively. The study included a total of 203 individuals, among which 100 were MI patients, while 103 were healthy controls. The mean age of MI patients was 57 years old, whereas that of the control group was 55 years old. Among the group of MI patients, 89 were male and 11 were female of whom $43 \%$ were smokers and
$57 \%$ non-smokers.

The study utilized multiplex $\mathrm{SNaPshot}$ assay to detect SNPs (or variants) that might be associated with MI. This early detection assay is needed to screen the individuals at high risk of the disease, considering the time factor and sensitivity of molecular diagnostic tools. The genotypes referring to the six genes were identified across the 203 participants and data is shown in Table 3. As a model, two typical GeneMapper electropherograms describing the most 
Table S1. Characteristics of subjects involved in the study in terms of numbers, gender, smoking habits

\begin{tabular}{|c|c|c|c|c|}
\hline \multirow{2}{*}{ Age } & Control & 103 & 55 & $54 \%$ \\
\hline & \multicolumn{2}{|c|}{ Gender } & \multicolumn{2}{|c|}{ Nationality } \\
\hline \multirow[t]{2}{*}{ Patients group (100) } & Male & Female & Male & Female \\
\hline & \multicolumn{2}{|c|}{$43(43 \%)$} & \multicolumn{2}{|c|}{57 (57\%) } \\
\hline
\end{tabular}

common patterns of healthy controls and MI patients are shown in Figure 1. The genotypes based on SNaPshot patterns of these two participants are GG/CA/CC/TT/AA/CC and GG/CC/CA/TT/AA/CT, referring to SNPs of the six genes LRP8, PCSK9, HNF1A, SVEP1, BRAP and HNF1A genes, respectively. Four out of the six synthesized SBE primers are reverse primers of the six genes. These four primers target sequences of the LRP8, PCSK9, HNF1A, and BRAP genes. Then, the actual genotypes of the two individuals in Figure 1 are CC/GT/GG/TT/TT/CC and CC/GG/GT/ TT/TT/CT. Based on the information available in the gene bank (https://www.ncbi.nlm.nih.gov), rare alleles of the six studied genes are complementary allele $\mathrm{T}$ (e.g., A allele), complementary allele $\mathrm{T}$ (e.g., A allele), complementary allele T (e.g., A allele), C, complementary allele C (e.g., G allele), and $\mathrm{T}$ allele, respectively. Interestingly, rare allele of variant rs111245230 (e.g., complementary allele T) was almost non-existent in the Saudi population, indicating this variant is di-allelic or harbor only two alleles (common and rare) that are conserved as an ethnic signature of the Saudi population, while harbor multiple alleles in populations with other ethnic backgrounds. Additionally, the variant rs3782886 was shown to be mono-allelic (e.g., complementary allele T) in the Saudi population that displayed only the homozygous genotype of complementary T allele (e.g., AA genotype) indicating complete absence of the rare allele in both homozygous or heterozygous conditions (Table 3). As per the association of the different variants and MI patients, the statistical analysis indicated that the rare allele of the rs11591147 variant (e.g., A allele) significantly is reduced $(P=.0013)$ in MI patients (rare allele frequency $=0.28$ ) as compared with that of the healthy controls (rare allele frequency $=0.41)($ Table 4$)$. The odd ratio $(\mathrm{OR})$, as a relative measure of effect, of the common allele was estimated as 1.85 with a confidence interval (CI) of 0.84-4.05 at $95 \%$ significance level. The OR value indicates that MI patients are much more likely to carry the common allele of PCSK9 gene than the healthy control. The confidence interval (CI) indicates the level of uncertainty around the measure of effect. CI was measured in the present study because we recruited a small sample of the overall Saudi population. Therefore, by having an upper and lower confidence limit, we can infer that the true population effect lies between these two points. The results of the two variants rs2259816 and rs2259820 also indicated reductions in the rare alleles (e.g., A and T, respectively), but not at the statistical level. Further analysis of the two variants with larger number of participants might indicate otherwise. Thus, we can confidently conclude that the nonappearance of the complementary allele $\mathrm{T}$ (e.g., A allele) of variant rs11591147 in a given individual is a strong risk factor of the occurrence of the MI disease.

\section{DISCUSSION}

Six SNPs or variants in the present study were selected on the basis of multiple recent large-scale genome wide association studies showing strong statistically significant association with MI risk. The main focus of the study was on investigating the association between the genetic markers rs5174, rs11591147, rs2259816, rs111245230, rs3782886 and rs2259820, referring to LRP8, CSK9, HNF1A, SVEP1, BRAP and HNF1A genes, respectively, and MI among Saudi patients. The mean age of MI patients in the present study was 57 years old. A similar study conducted by Kathiresan et al showed that the majority of the MI cases were observed among the individuals who are $>65$ years old [Kathiresan 2009]. Another study conducted by Maas et al showed that cardiovascular disease develops 7-10 years early among men as compared to women [Maas 2010]; however, it still is the major cause of death in women over the age of 65 years. The genotyping method used in the present study is SnapShot assay that assisted in screening the six SNPs, simultaneously with single reaction in individual samples. Similar to the present study, Ben et al determined the genotyping of two genes, CYP2D6 and ADRB1, by using SNaPshot assay [Ben 2016]. The results showed that this technique is simple, efficient, and accurate in detecting the genotypes based on these two genes in significantly reduced costs. Another study conducted by Ghosh et al found that $\mathrm{SNaPshot} \mathrm{technique} \mathrm{is} \mathrm{an}$ informative approach deeply helpful in detecting parent- and yet stage-of-origin of nondisjunction and underscores the need for characterizing additional markers [Ghosh 2012].

The output of 40 random samples genotyped by $\mathrm{SNaPshot}$ method was validated by Sanger sequencing (data not shown). Moreover, the study achieved an agreement of $100 \%$ with $\mathrm{SNaPshot}$ for the six SNP analyzed in these 40 samples. In a similar context, significant association with $\mathrm{CAD}$ and $\mathrm{MI}$ was 
Table S2. Prior information on relation of single nucleotide polymorphisms (SNPs) to CAD

\begin{tabular}{lcc}
\hline SNP number & Gene name & Correlation with CAD \\
\hline rs5174 & LRP8 & Lieb et al. (2008) \\
rs11591147 & PCSK9 & Zaimkohan et al. (2015) \\
rs2259816 & HNF1A & Kleber et al. (2010) \\
rs111245230 & SVEP1 & Nelson et al. (2017) \\
rs3782886 & BRAP & Ozaki et al. (2016) \\
rs2259820 & HNF1A & Zhou et al. (2017)
\end{tabular}

observed by Shen et al [Shen 2007]. Moreover, permutation testing showed significant $\mathrm{P}$-value for the association between SNP R952Q and CAD or MI. The results by Shen et al also stated that homozygous carriers of the ApoER2-R952Q variant have a twofold increased risk of these conditions [Shen 2007]. In addition, Lieb et al found lack of association of the LRP8 R952Q polymorphism with sporadic CAD/MI [Lieb 2008]. There was no evidence for association of this variant with familial MI/CAD in the so far largest sample of familial MI/CAD cases.

In the present study, allele frequencies of the PCSK9 gene (C: 0.59 and T: 0.41) in Saudi control samples were very much similar to those of the European populations (C: 0.60 and T: 0.40), while both are completely different from those of East Asian populations (C: 0.97 and T: 0.03). However, previous studies have shown that PCSK9 R46L carriers have lower levels of lipoprotein and LDL cholesterol, along with reduced risk of aortic valve stenosis and myocardial infarction [Cameron 2006; Kathiresan 2008]. Another study conducted by Almontashiri et al showed that the decreased level of LDL-C attained by statins is associated with only a $25 \%$ reduction in the risk of coronary events, which was also confirmed in European ancestry groups [Almontashiri 2014]. The global data in 1000-genome project from different ethnic groups was significantly affected on the basis of minor (or rare) allele frequencies in the present study.

The results of present study have shown significant difference between rare allele frequencies of SNP rs11591147 referring to PCSK9 gene between $\mathrm{MI}$ patients and controls. This indicates that the A allele of rs11591147 variant can be a protective allele, thus, is associated with the decreased risk of MI in Saudi individuals. A similar study conducted by Guella et al showed significant association between rs11591147 carriers and lower LDL-C levels [Guella 2010]. Another study conducted by Zaimkohan et al in Iran showed insignificant association between the R46L and lower risk of MI [Zaimkohan 2015]. However, SNPs of rs3782886 and rs2259820 in the present study were synonymous and benign (SIFT/Polyphen) showing no significant differences between patients and control groups albeit the locus of rs3782886 variant in the Saudi population is mono-allelic (e.g., T allele only) displayed only the homozygous genotype of TT.

The study results were limited because of the small sample size that could affect the reliability of statistical analysis and working in a single center. Moreover, the SNaPShot technique used in this study is not capable of detecting novel allele presented in sample location; instead, it indicated the nonexistence of rare alleles in the three variants rs 11591147 (e.g., A allele), rs2259816 (e.g., A allele), and rs3782886 (e.g., $\mathrm{C}$ allele). Difficulties were faced in SNPs selection, based on different ethnic groups due to lack of local variants database in the target region that caused hindrance in conducting the association studies.

\section{CONCLUSION}

The present study has examined the association between $\mathrm{MI}$ and SNPs of six genes related to the disease. It has also evaluated the gene variants as genetic risk factors to MI among patients in Saudi Arabia. The principal finding of the study is SNP rs11591147 working as a protective allele against the MI disease in Saudi Arabia, which means that the rare allele (e.g., A) frequency of this SNP was significantly higher in the healthy control individuals and lower among the patients group. The results also showed that Multiplex $\mathrm{SNaPShot}$ assay is a rapid, reliable, flexible, accurate technique and cost-effective to detect risk alleles of any complex diseases in the Kingdom of Saudi Arabia or in any other ethnic groups. However, the study results recommend conducting future studies with a larger sample size and multi centers for reliable statistical analysis and more significant results. It also recommends the creation of local genomic variants database from a healthy Saudi population that will give local distribution for the selection of variants in such association studies for any complex diseases such as MI, celiac, obesity, or diabetes.

\section{ACKNOWLEDGEMENTS}

This project was funded by the Deanship of Scientific Research (DSR) at King Abdulaziz University, Jeddah, under grant no. (1-287-36/HiCi). The Authors, therefore, acknowledge with thanks DSR for technical and financial support.

\section{REFERENCES}

Almontashiri NAM, Vilmundarson RO, Ghasemzadeh N, Dandona S, Roberts R, Quyyumi AA, Stewart AFR. 2014. Plasma PCSK9 levels are elevated with acute myocardial infarction in two independent retrospective angiographic studies. PLoS One, 9(9), e106294.

Ben S, Cooper-DeHoff RM, Flaten HK, Evero O, Ferrara TM, Spritz RA, Monte AA. 2016. Multiplex SNaPshot-a new simple and efficient CYP2D6 and ADRB1 genotyping method. Human Genomics, 10(1), 11.

Cameron J, Holla ØL, Ranheim T, Kulseth MA, Berge KE, Leren TP. 2006. Effect of mutations in the PCSK9 gene on the cell surface LDL receptors. Human Molecular Genetics, 15(9), 1551-1558.

Ferreira LE, Secolin R, Lopes-Cendes I, Cabral NL, de França PHC. 2019. Association and interaction of genetic variants with occurrence of ischemic stroke among Brazilian patients. Gene. 
Ghosh D, Gochhait S, Banerjee D, Chatterjee A, Sinha S, Nandagopal K. 2012. SNaPshot assay in quantitative detection of allelic nondisjunction in Down syndrome. Genetic Testing and Molecular Biomarkers, 16(10), 1226-1235.

Guella I, Asselta R, Ardissino D, Merlini PA, Peyvandi F, Kathiresan S, Duga S. 2010. Effects of PCSK9 genetic variants on plasma LDL cholesterol levels and risk of premature myocardial infarction in the Italian population. Journal of Lipid Research, 51(11), 3342-3349.

Isordia-Salas I, Alvarado-Moreno JA, Jiménez-Alvarado RM, Hernández-Juárez J, Santiago-Germán D, Leaños-Miranda A, Majluf-Cruz A. 2018. Association of renin-angiotensin system genes polymorphisms and risk of premature ST elevation myocardial infarction in young Mexican population. Blood Coagulation \& Fibrinolysis, 29(3), 267-274.

Kashyap S, Kumar S, Agarwal V, Misra DP, Rai MK, Kapoor A. 2018. The association of polymorphic variants, rs2267788, rs1333049 and rs 2383207 with coronary artery disease, its severity and presentation in North Indian population. Gene, 648, 89-96.

Kathiresan S, Voight BF, Purcell S, Musunuru K, Ardissino D, Mannucci PM, Schunkert H. 2009. Genome-wide association of early-onset myocardial infarction with single nucleotide polymorphisms and copy number variants. Nature Genetics, 41(3), 334-341.

Kleber ME, Grammer TB, Renner W, März W. 2010. Effect of the rs2259816 polymorphism in the HNF1A gene on circulating levels of c-reactive protein and coronary artery disease (the ludwigshafen risk and cardiovascular health study). BMC medical genetics, 11(1), 157.

Li Y, Xu X, Zhang D, Cheng W, Zhang Y, Yu B, Zhang Y. 2019. Genetic variation in the leukotriene pathway is associated with myocardial infarction in the Chinese population. Lipids in health and disease, 18(1), 25.

Lieb W, Zeller T, Mangino M, Götz A, Braund P, Wenzel JJ, Bruse P. 2008. Lack of association of genetic variants in the LRP8 gene with familial and sporadic myocardial infarction. Journal of molecular medicine, 86(10), 1163-1170.

Ma WQ, Wang Y, Han XQ, Zhu Y, Liu NF. 2018. Associations between LPL gene polymorphisms and coronary artery disease: evidence based on an updated and cumulative meta-analysis. Bioscience reports, 38(2), BSR20171642.
Maas A, Appelman YEA. 2010. Gender differences in coronary heart disease. Netherlands Heart Journal, 18(12), 598-603.

Matsuoka R, Abe S, Tokoro F, Arai M, Noda T, Watanabe S, Kato K. 2015. Association of six genetic variants with myocardial infarction. International journal of molecular medicine, 35(5), 1451-1459.

Nelson CP, Goel A, Butterworth AS, Kanoni S, Webb TR, Marouli E, Giannakopoulou O. 2017. Association analyses based on false discovery rate implicate new loci for coronary artery disease. Nature genetics, 49(9), 1385.

Nikpay M, Goel A, Won HH, Hall LM, Willenborg C, Kanoni S, Webb TR. 2015. A comprehensive 1000 Genomes-based genome-wide association meta-analysis of coronary artery disease. Nature genetics, 47(10), 1121.

Ozaki, Kouichi, and Toshihiro T. 2016. Molecular genetics of coronary artery disease. Journal of human genetics 61(1), 71.

Shen GQ, Li L, Girelli D, Seidelmann SB, Rao S, Fan C, Hu Y. 2007. An LRP8 variant is associated with familial and premature coronary artery disease and myocardial infarction. The American Journal of Human Genetics, 81(4), 780-791.

Wang Y, Wang L, Liu X, Zhang Y, Yu L, Zhang F, Wang X. 2014. Genetic variants associated with myocardial infarction and the risk factors in Chinese population. PloS one, 9(1), e86332.

Yasukochi Y, Sakuma J, Takeuchi I, Kato K, Oguri M, Fujimaki T, Yamada Y. 2018. Six novel susceptibility loci for coronary artery disease and cerebral infarction identified by longitudinal exome-wide association studies in a Japanese population. Biomedical reports, 9(2), 123-134.

Zaimkohan H, Keramatipour M, Ghaderian JT, Bazzaz AT, Piryaei M, Ghahhari NM, Ahani M. 2015. PCSK9 SNP RS11591147 association study with coronary artery disease risk in iran. Acta medica mediterranea, $31,1435-1438$.

Zhou YJ, Yin RX, Hong SC, Yang Q, Cao XL, Chen WX. 2017. Association of the HNF1A polymorphisms and serum lipid traits, the risk of coronary artery disease and ischemic stroke. The journal of gene medicine, 19(1-2), e2941. 